\title{
Lomustine Loaded Chitosan Nanoparticles: Characterization and in-Vitro Cytotoxicity on Human Lung Cancer Cell Line L132
}

\author{
Archana Mehrotra, ${ }^{a}$ Ramesh Chand Nagarwal, ${ }^{b}$ and Jayanta Kumar Pandit ${ }^{*}, b$ \\ ${ }^{a}$ NRI Institute of Pharmaceutical Sciences; Bhopal-462022 (M.P), India: and ${ }^{b}$ Department of Pharmaceutics, Institute of \\ Technology, Banaras Hindu University; Varanasi-221005, India. \\ Received August 15, 2010; accepted December 15, 2010; published online December 24, 2010
}

The aim of this work was to prepare chitosan nanoparticles loaded with antineoplastic drug Lomustine (LCNPs), by ionic-gelation method with homogenization. The nanoparticles were characterized for particle size, polydispersity index (PDI), surface morphology, encapsulation efficiency, in-vitro drug release and cytotoxicity on human lung cancer cell line L132 by 3-(4,5-dimethylthiazol-2-yl)-2,5-diphenyltetrazolium bromide (MTT) assay. The particle size, zeta potential and encapsulation efficiency of prepared nanoparticles ranged from $75 \pm 1.1$ to $637 \pm 1.6 \mathrm{~nm}$ (PDI from $0.05 \pm 0.001$ to $0.18 \pm 0.007$ ), $37.2 \pm 0.21$ to $53.8 \pm 0.18 \mathrm{mV}$ and $66.74 \pm 1.4$ to $98.0 \pm 1.8 \%$ respectively. The particles were spherical in shape with smooth surface in scanning electron microscopy (SEM) and transmission electron microscopy (TEM) images. Mechanical shearing by homogenization treatment significantly changed the nanoparticle size. The drug release rate was biphasic and diffusion controlled over the 8 h. LCNPs greatly inhibited the growth of the $\mathbf{L} 132$ cancer cell line used in this study in comparison to the native Lomustine (LMT).

Key words Lomustine; chitosan; nanoparticle; in-vitro cytotoxicity

Lomustine (LMT) is a nitrosourea of compound, mainly used in brain tumors, resistant or relapsed Hodgkin's disease, other lymphomas, lung cancer, malignant melanoma and various solid tumors. LMT is used either alone or in combination with other cytostatic drugs. Unfortunately, it also has serious untoward side effects and is rather a toxic compound, and the therapeutic index, the ratio between the toxic dose and the therapeutic dose, is not very favorable. Potential benefits of biodegradable polymeric nanoparticles have attracted considerable attention as colloidal drug delivery. Nanoparticles can be transported via the circulation to different body sites. Chitosan nanoparticles (NPs) have been investigated for targeted delivery to the colon, mucosa in cancer therapy for vaccine delivery, and gene delivery. Additionally, such systems have the ability to control the rate of drug administration, thus controlling the duration of the therapeutic effect, and also deliver the drug to specific sites. ${ }^{1-6)}$

The ionotropic gelation method has already been established to prepare chitosan nanoparticles that uses protonized $-\mathrm{NH}_{3}^{+}$to interact with an anion such as tripolyphosphate. ${ }^{7} \mathrm{In}$ addition, reversible physical crosslinking by electrostatic interaction, instead of chemical crosslinking, is applied to prevent possible toxicity of reagents and other undesirable effects. ${ }^{89}$ To decrease the size variation and polydispersity of nanoparticles, ultrasonication at increasing reaction time or radiation amplitude has been used. Tsai et al. ${ }^{10)}$ prepared chitosan nanoparticles by modified ionic gelation with mechanical stirring with ultrasonication. Other means to increase the shearing effects are high pressure homogenization instead stirring.

As a non-ionic hydrophilic polymer, polyethylene glycol (PEG) exhibits outstanding protein resistance, minimal toxicity and immunogenicity. PEG coated nanoparticles have been found to be of great potential in therapeutic application for controlled release of drugs and site-specific drug delivery. ${ }^{11)}$ PEG chains that have attached to the surface or formed the corona of a nanoparticle exhibit rapid motion in aqueous media and have a large excluded volume, and steric repulsion results from a loss of configurational entropy of the bound PEG chains. ${ }^{12)}$ In addition, the hydrophilic PEG can form a hydrated outer shell, which protects the nanoparticles from being quickly uptaken by the reticuloendothelial system extending the half-life of drugs, and altering their tissue distribution. ${ }^{13)}$

This study aims to develop a LMT loaded chitosan nanoparticles (LCNPs) by ionic gelation method with homogenization to increase the antineoplasitc efficiency of LMT. Particle size and morphology of the fabricated nanoparticles were studied by DLS, SEM and TEM. Effect of various parameters such as chitosan $(\mathrm{CH}) /$ tripolyphosphate (TPP) ratio, PEG coating, and homogenization on nanoparticle size and encapsulation were studied. The interaction between LMT and PEG-chitosan and in-vitro release behavior of LMT loaded chitosan nanoparticles and PEG coated LCNPs were studied. Finally, the cytostatic effect of LMT-loaded nanoparticles was studied in human lung cancer cell line L132.

\section{Experimental}

Chitosan (CH) (deacetylation degree $85 \%$ ) was obtained as a gift sample from Marine Chemicals (Cochin, Kerala, India). Sodium tripolyphosphate (TPP) of analytical grade was obtained as a gift sample from Devdhar chemicals (Pune, India). Lomustine (LMT) pharmaceutical grade was obtained from Fujian Provincial Medicines and Health Products, Xiamen Import and Export Corporation (China). All other chemicals were of analytical grade and used as received.

Preparation of Nanoparticles The nanoparticles were prepared by modification of ionic gelation method. ${ }^{14)}$ The basic formulation (formulation batch LC1 was prepared as follows: appropriate quantity of chitosan was dissolved in $1.5 \% \mathrm{v} / \mathrm{v}$ aqueous acetic acid solution under magnetic stirring. Then $10 \% \mathrm{w} / \mathrm{w}$ LMT was added to this solution with homogenization at $11000 \mathrm{rpm}$ for $20 \mathrm{~min}$, and then TPP was added and the solution was further homogenized. Afterwards, the solvent was evaporated in a rotary evaporator under atmospheric pressure. The PEG coated drug loaded nanoparticles (DNPs) (formulation batch LCP1) were prepared as per the same method of basic formulation with PEG $6000(20 \mathrm{mg} / \mathrm{ml})$ added to the chitosan solution by homogenization at $11000 \mathrm{rpm}$ for $15 \mathrm{~min}$ before addition of LMT. PEG coated CH Nanoparticles loaded LMT were separated by cooling centrifugation at $19400 \times \mathbf{g}$. For the formulations LCH1 and LCH2 the processing was done at homogenization speed of $13000 \mathrm{rpm}$ and $22000 \mathrm{rpm}$ respectively (Table 1). 
Particle Size, Zeta Potential and Morphology The particle size, size distribution and zeta potential of the fabricated chitosan nanoparticles were measured in a Zetasizer (Malvern Instruments DTS Ver 4.10). Shape and surface morphology were determined by transmission electron microscopy (TEM) using FEI Morgagni $268 \mathrm{D}$ instrument at an accelerating voltage of $120 \mathrm{kV}$. A drop of aqueous solution of sample was placed on a membrane coated grid surface. A drop of $1 \%$ phosphotungstic acid was immediately added to the surface of the grid. After 1 min excess fluid was removed and the grid surface was air dried at room temperature before being loaded for TEM. For scanning electron microscopy (SEM) a thin film of aqueous dispersion of nanoparticles was applied on double stick tape over an aluminium stub and air dried to get uniform layer of particles. These particles were coated with gold using sputter gold coater, and subjected to SEM on Leo 435 VP, Cambridge, U.K.

Fourier Transform (FT)-IR Analysis LCNPs and blank NPs were separated by centrifugation of the nanosuspension and freeze dried. FT-IR spectra were obtained using a $\mathrm{KBr}$ pellet in FT-IR spectrophotometer (Shimadzu8400 S, Japan). \% Transmittance (\%T) was recorded in the spectral region of $500-4500 \mathrm{~cm}^{-1}$ using a resolution of $4 \mathrm{~cm}^{-1}$ and 40 scans

Encapsulation Efficiency Appropriate amount of freeze dried LCNPs was resuspended in phosphate buffer $\mathrm{pH} 7.4$ and digested with minimum amount of $95 \% \mathrm{v} / \mathrm{v}$ ethanol until complete material was dissolved. The digested homogenate was centrifuged at $19400 \times \boldsymbol{g}$ (cooling centrifuge, REMI) for $30 \mathrm{~min}$ and the supernatant was analyzed for drug entrapment. The LMT entrapment was measured using shimadzu 1601 UV/VIS spectrophotometer at $230 \mathrm{~nm}$. The encapsulation efficiency was calculated using equation:

$$
\% \text { encapsulation efficiency }=\frac{\text { total mass of drug in nanoparticles }}{\text { mass of drug used in the formulation }} \times 100
$$

In-Vitro Drug Release Studies LMT release from different LCNPs was determined using dialysis-bag diffusion technique under magnetic stirring. LCNPs $(25 \mathrm{mg}$ ) were redispersed in $3 \mathrm{ml}$ phosphate buffer saline (PBS) solution $\mathrm{pH} 7.4$ and placed in a dialysis membrane bag with a molecular cutoff of $12 \mathrm{kDa}$, tied and placed into $150 \mathrm{ml}$ PBS solution in a beaker. The entire system was kept at $37^{\circ} \mathrm{C}$ with continuous magnetic stirring on a thermostatically controlled magnetic stirrer. At appropriate time intervals, $3 \mathrm{ml}$ of release medium was removed and the same volume of fresh PBS solution was added into the system. The amount of LMT in the release medium was evaluated by dilution with ethanol $95 \% \mathrm{w} / \mathrm{v}$ by UV spectrophotometer at $230 \mathrm{~nm}$.

Cytotoxic Activity Human lung cancer cell line L132 was obtained from cell bank of National Centre for Cell Sciences (NCCS) Pune, India, for studying the antiproliferative action of pure drug and the LCNPs were tested on human lung cancer cell line L132. L132 cells were seeded at a density of $1 \times 10^{4}$ cells/well in $180 \mu \mathrm{l}$ growth medium RPMI-1640 in 96-well plates and incubated for $24 \mathrm{~h}$ prior to the addition of $20 \mu \mathrm{l}$ solutions of LMT, or resuspended LCNPs, and PEG coated LCNPs in phosphate buffer pH 7.4 with final concentrations of $20,50,75$ and $100 \mu \mathrm{g} / \mathrm{ml}$, respectively. Cells were incubated for $12,24,36$ and $48 \mathrm{~h}$ before the addition of 3-[4,5-dimethylthiazol-2-yl]-3,5-diphenyltetrazolium bromide dye (MTT $20 \mu \mathrm{l} /$ well) at a concentration of $5 \mathrm{mg} / \mathrm{ml}$ in PBS buffer). After further incubation of $4 \mathrm{~h}$, the media was removed and replaced with $150 \mu \mathrm{l}$ dimethyl sulfoxide (DMSO). The absorbance was read at $570 \mathrm{~nm}$ in a microplate reader following a 300-s auto mixing. The cell viability (\%) was calculated according to the following equation:

cell viability $(\%)=($ OD570 $($ sample $) /$ OD570 $($ control $)) \times 100$

Where, OD570 (sample) represents measurement from the wells treated with samples and OD570 (control) from the wells treated with RPMI 1640 media only.

Statistical Analysis Results are given as mean \pm S.D. Mean values of nanoparticle size, polydispersity index, zeta potential and encapsulation efficiency were compared using the Student's $t$-test. Differences are considered significant at a level of $p<0.05$. In-vitro data were analyzed with a one-way ANOVA. Critical value of $F$ was considered at $5 \%$ level of significance.

\section{Results}

Particle Size, Zeta Potential and Morphology The mean particle sizes and PDI of LCNPs are shown in Table 1. For the basic formulation (LC1) nanoparticle size and polydispersity index (PDI) were observed to be $98 \pm 0.9 \mathrm{~nm}$ and $0.12 \pm 0.002 \mathrm{~nm}$ respectively. On PEG coating (batch LCP1) particle size was increased up to $637 \pm 1.6 \mathrm{~nm}$. Further change in PEG concentration (batches LCP2 to LCP5) showed increase in size with concentration of PEG. With increasing concentration of chitosan (batch LC1 to LC5) particle size increased significantly $(p<0.05)$ from $119 \pm 0.4$ to $338 \pm 0.6 \mathrm{~nm}$ but the polydispersity was decreased from $0.076 \pm 0.006$ to $0.062 \pm 0.008$. Increased acetic acid concentration (batch LC8 to LC11) resulted in decreased particle size. Particle size was increased significantly $(p<0.05)$ from $152 \pm 1.6$ to $549 \pm 1.1 \mathrm{~nm}$ when TPP ratio was increased (batches LC1, LC12 to LC14) and PDI was increased from $0.082 \pm 0.013$ to $0.14 \pm 0.005$. Increasing the amount of LMT (batches LC6 and LC7) resulted in increased particle size from $207 \pm 1.2$ to $349 \pm 1.6 \mathrm{~nm}$ and PDI was increased from $0.15 \pm 0.011$ to $0.18 \pm 0.007$. A significant $(p<0.05)$ decrease in the particle size was observed when homogenization speed was increased (batch LCH1 and LCH2) with corresponding decrease in PDI.

Zeta potential of the nanoparticles of batch $\mathrm{LC} 1$ was $49.3 \pm 0.23 \mathrm{mV}$. It was decreased to $48.0 \pm 0.16 \mathrm{mV}$ on coating with PEG (batch LCP1) and further from 47.6 \pm 0.19 to $32.4 \pm 0.11 \mathrm{mV}$ (batch LCP2 to LCP5). Positive zeta potential value was observed for all batches. A significant difference $(p<0.01$ : Dunnett Multiple Comparisons Test) in zeta potential were observed in formulation batches $\mathrm{LC} 12$ to $\mathrm{LC} 15$, when CH:TPP ratio was increased (from $50.1 \pm 0.09$ to $53.8 \pm 0.18 \mathrm{mV}$ whereas changes in zeta potential with formulation batches LCH1 and LCH2 with increasing homogenization speed were not significant $(p>0.05)$. Zeta potential was decreased for batches LC1to LC5 with increasing chitosan concentration from $49.3 \pm 0.23$ to $40.8 \pm 0.15 \mathrm{mV}$. The incorporation of LMT in the NPs had a pronounced effect on particle size and led to a significant decrease $(p<0.05)$ in the zeta potential, reaching a value around $+45.1 \mathrm{mV}$. Considering that LMT was dispersed in $1.5 \% \mathrm{v} / \mathrm{v}$ acetic acid $(\mathrm{pH} c a$. $4.0)$ it resulted in a positive charge.

The LCNPs were analyzed by TEM (Figs. 1C,D) and SEM (Figs. 1A, B) micrographs and found to be spherical in shape. The nanoparticle size, as observed by TEM and SEM did not correlate well with the size measured by Zetasizer.

Drug and Polymer Interaction Study by FT-IR The FT-IR spectra of LMT (Fig. 2) showed the characteristic peaks of cyclohexyl ring (at $1535 \mathrm{~cm}^{-1}$ and $1489 \mathrm{~cm}^{-1}$ ), $\mathrm{N}-\mathrm{O}-\mathrm{N}$ linkage (at $1076 \mathrm{~cm}^{-1}$ ) and $\mathrm{C}-\mathrm{Cl}$ stretch ( at $890 \mathrm{~cm}^{-1}$ ). In the spectrum of LCNPs (batch LC1) the characteristic peak of LMT was shifted to $1558 \mathrm{~cm}^{-1}, 1443 \mathrm{~cm}^{-1}$ and a peak at $1076 \mathrm{~cm}^{-1}$ was broadened. In the spectrum of PEG coated LCNPs (LCP1) a peak of LMT at $1076 \mathrm{~cm}^{-1}$ was not observed and a new peak at $1377 \mathrm{~cm}^{-1}$ was observed. Also the cyclohexyl ring absorption was shifted to $1567 \mathrm{~cm}^{-1}$ and $1444 \mathrm{~cm}^{-1}$. It was observed that LMT was included in the nanoparticles with physical crosslinking in both uncoated LCNPs and in PEG coated LCNPs by van der Wall's interaction, instead of chemical crosslinking.

Drug Encapsulation Efficiency The encapsulation efficiency was found to be directly proportional to the preparative parameters tested (Table 1). Encapsulation efficiency was significantly $(p<0.05)$ affected by chitosan concentration (LC1 to LC5), $\mathrm{CH}$ : TPP mass ratio (LC12 to LC15) and LMT (LC6-LC7). It ranged from $66.74 \pm 1.4$ to $98.0 \pm 1.8$, $77.2 \pm 1.2$ to $83.8 \pm 1.1$ and $79.3 \pm 1.3$ to $82 \pm 2.1$ respectively. 
Encapsulation efficiencies were increased with increase in concentration of PEG (batches LCP1 to LCP5).

In-Vitro Release Study The LCNPs showed burst release followed by release upto $6 \mathrm{~h}$. After $6 \mathrm{~h}$ of dialysis in PBS ( $\mathrm{pH} 7.4$ ), 85.39\% and $78.64 \%$ of LMT was released from batches LC1 and LCP1 respectively and further decreased with increasing concentration of PEG (Fig. 3D). As expected the release of LMT was extended with increasing concentration of $\mathrm{CH}$ with $t_{50 \%}$ values from 3.0 to $5.0 \mathrm{~h}$ for batches LC1 to LC5 (Fig. 3A). Similar drug release profiles were observed for other batches LC12 to LC15 with increasing $\mathrm{CH}$ : TPP ratio (Fig. 3C) and LC6 - LC7 with increasing concentration of LMT. Release was significantly affected $(p<0.05)$ in batches (LC12-LC15). The increase in acetic acid concentration (LC1, LC8- LC11) resulted in faster
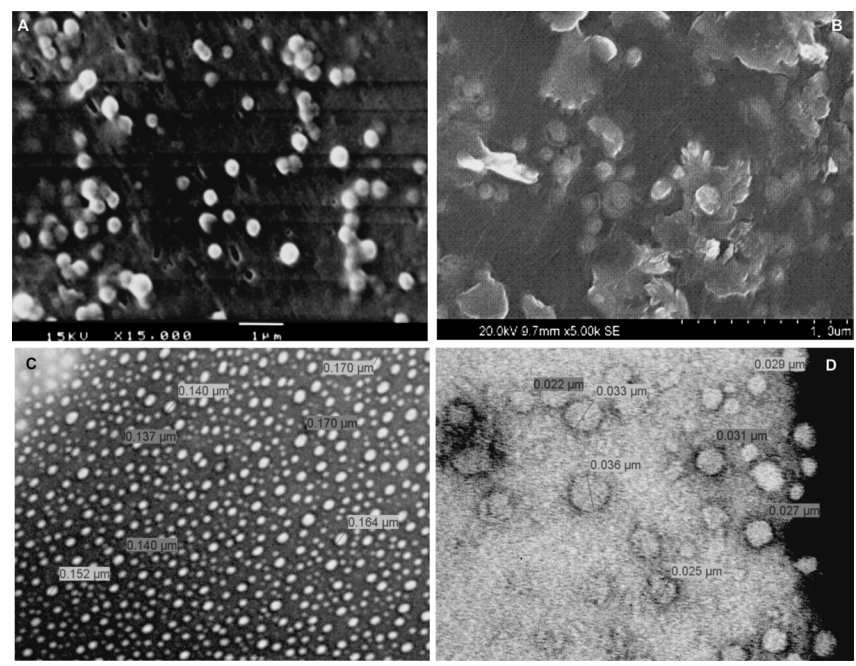

Fig. 1. SEM Micro Graphs (A) LMT Loaded Chitosan Nanoparticles (B) PEG Coated LCNPs; TEM Micrograph (C) LMT Loaded Chitosan Nanoparticles (D) PEG Coated LCNPs

Table 1. Formulation Composition, Particle Size, Zeta Potential, and \% EE

\begin{tabular}{|c|c|c|c|c|c|c|c|c|c|c|}
\hline $\begin{array}{l}\text { S. } \\
\text { No. }\end{array}$ & $\begin{array}{l}\text { Batch } \\
\text { code }\end{array}$ & $\begin{array}{c}\mathrm{CH} \\
(\mathrm{mg} / \mathrm{ml})\end{array}$ & $\begin{array}{c}\text { AA } \\
\%\end{array}$ & $\begin{array}{c}\mathrm{LMT} \\
\%\end{array}$ & $\begin{array}{l}\text { TPP } \\
\text { ratio }\end{array}$ & $\begin{array}{c}\text { PEG } \\
(\mathrm{mg} / \mathrm{ml})\end{array}$ & $\begin{array}{c}\text { PS (nm) } \\
(\text { mean土S.D.*) }\end{array}$ & $\begin{array}{c}\text { PDI } \\
(\text { mean } \pm \text { S.D.*) }\end{array}$ & $\begin{array}{c}\mathrm{ZP}(\mathrm{mV}) \\
(\text { mean土S.D.*) }\end{array}$ & $\begin{array}{c}\% \mathrm{EE} \\
(\text { mean } \pm \text { S.D.*) }\end{array}$ \\
\hline 1 & LC1 & 1.0 & 1.5 & 10 & $3: 1$ & - & $98 \pm 0.9$ & $0.12 \pm 0.002$ & $49.3 \pm 0.23$ & $66.74 \pm 1.4$ \\
\hline 2 & LC2 & 1.5 & 1.5 & 10 & $3: 1$ & - & $119 \pm 0.4$ & $0.076 \pm 0.001$ & $46.9 \pm 0.18$ & $80 \pm 2.6$ \\
\hline 3 & LC3 & 2.0 & 1.5 & 10 & $3: 1$ & - & $254 \pm 0.8$ & $0.070 \pm 0.006$ & $43.8 \pm 0.14$ & $93.33 \pm 1.3$ \\
\hline 4 & LC4 & 2.5 & 1.5 & 10 & $3: 1$ & - & $286 \pm 1.1$ & $0.069 \pm 0.007$ & $41.3 \pm 0.08$ & $96.2 \pm 2.3$ \\
\hline 5 & LC5 & 3.0 & 1.5 & 10 & $3: 1$ & - & $338 \pm 0.6$ & $0.062 \pm 0.008$ & $40.8 \pm 0.15$ & $98.0 \pm 1.8$ \\
\hline 6 & LC6 & 1.0 & 1.5 & 25 & $3: 1$ & - & $207 \pm 1.2$ & $0.15 \pm 0.011$ & $46.5 \pm 0.15$ & $79.3 \pm 1.3$ \\
\hline 7 & LC7 & 1.0 & 1.5 & 50 & $3: 1$ & - & $349 \pm 1.6$ & $0.18 \pm 0.007$ & $45.1 \pm 0.16$ & $82.0 \pm 2.1$ \\
\hline 8 & LC8 & 1.0 & 3.0 & 10 & $3: 1$ & - & $96 \pm 1.5$ & $0.1 \pm 0.011$ & $44.6 \pm 0.14$ & $83.2 \pm 1.2$ \\
\hline 9 & LC9 & 1.0 & 4.0 & 10 & $3: 1$ & - & $92 \pm 1.3$ & $0.14 \pm 0.020$ & $42.8 \pm 0.16$ & $86.4 \pm 2.4$ \\
\hline 10 & LC10 & 1.0 & 5.0 & 10 & $3: 1$ & - & $90 \pm 0.7$ & $0.07 \pm 0.006$ & $39.7 \pm 0.13$ & $88 \pm 0.9$ \\
\hline 11 & LC11 & 1.0 & 6.0 & 10 & $3: 1$ & - & $82 \pm 1.2$ & $0.082 \pm 0.013$ & $37.2 \pm 0.21$ & $88.7 \pm 1.1$ \\
\hline 12 & LC12 & 1.0 & 1.5 & 10 & $4: 1$ & - & $152 \pm 1.6$ & $0.083 \pm 0.023$ & $50.1 \pm 0.09$ & $77.2 \pm 1.2$ \\
\hline 13 & LC13 & 1.0 & 1.5 & 10 & $5: 1$ & - & $260 \pm 1.2$ & $0.089 \pm 0.014$ & $52.0 \pm 0.15$ & $80.0 \pm 1.6$ \\
\hline 14 & LC14 & 1.0 & 1.5 & 10 & $6: 1$ & - & $446 \pm 0.8$ & $0.1 \pm 0.007$ & $53.2 \pm 0.21$ & $81.2 \pm 0.9$ \\
\hline 15 & LC15 & 1.0 & 1.5 & 10 & $7: 1$ & - & $549 \pm 1.1$ & $0.14 \pm 0.005$ & $53.8 \pm 0.18$ & $83.8 \pm 1.1$ \\
\hline 16 & LCP1 & 1.0 & 1.5 & 10 & $3: 1$ & 10 & $543 \pm 1.3$ & $0.1 \pm 0.002$ & $48.0 \pm 0.16$ & $69.7 \pm 1.2$ \\
\hline 17 & LCP2 & 1.0 & 1.5 & 10 & $3: 1$ & 20 & $637 \pm 1.6$ & $0.16 \pm 0.002$ & $47.6 \pm 0.19$ & $73.33 \pm 1.8$ \\
\hline 18 & LCP3 & 1.0 & 1.5 & 10 & $3: 1$ & 30 & $679 \pm 0.2$ & $0.19 \pm 0.005$ & $45.2 \pm 0.13$ & $77.31 \pm 0.8$ \\
\hline 19 & LCP4 & 1.0 & 1.5 & 10 & $3: 1$ & 40 & $715 \pm 1.2$ & $0.17 \pm 0.016$ & $41.8 \pm 0.08$ & $81.67 \pm 1.1$ \\
\hline 20 & LCP5 & 1.0 & 1.5 & 10 & $3: 1$ & 50 & $762 \pm 0.9$ & $0.19 \pm 0.008$ & $32.4 \pm 0.11$ & $84.10 \pm 0.9$ \\
\hline 21 & LCH1 & 1.0 & 1.5 & 10 & $3: 1$ & & $81 \pm 0.6$ & $0.07 \pm 0.003$ & $49.2 \pm 0.14$ & $77.0 \pm 2.5$ \\
\hline 22 & LCH2 & 1.0 & 1.5 & 10 & $3: 1$ & & $75 \pm 1.1$ & $0.05 \pm 0.001$ & $49.0 \pm 0.18$ & $77.5 \pm 0.7$ \\
\hline
\end{tabular}

CH: chitosan, TPP: sodium tripolyphosphate, AA: acetic acid, LMT: Lomustine, PS: particle size, ZP: zeta potential, EE: encapsulation efficiency, PEG: polyethyleneglycol, LCNPs: Lomustine loaded chitosan nanoparticles, NPs: blank chitosan nanoparticles. * S.D.: Standard deviation. drug release. The $t_{50 \%}$ was $2.81,2.65,2.44$ and $1.76 \mathrm{~h}$ for batches LC2-LC5 respectively (Fig. 3A). Similar results were obtained for batches LCH1 and LCH2. Effect of acetic acid (AA) concentration on in vitro release was also tested (Fig. 3B). One and a half percent AA concentration was found suitable. Release kinetics was evaluated by fitting obtained data into first order, zero order and Higuchi equations. Based on the results, LMT release from LCNPs followed Higuchi equation.

Cytotoxicity Study Cytotoxic activity of native LMT and LCNPs formulation batches LC1 and LCP1 (Fig. 4) were studied. Viability of L132 cells was significantly reduced with LCNPs (batch LC1). Marked time dependent inhibitory

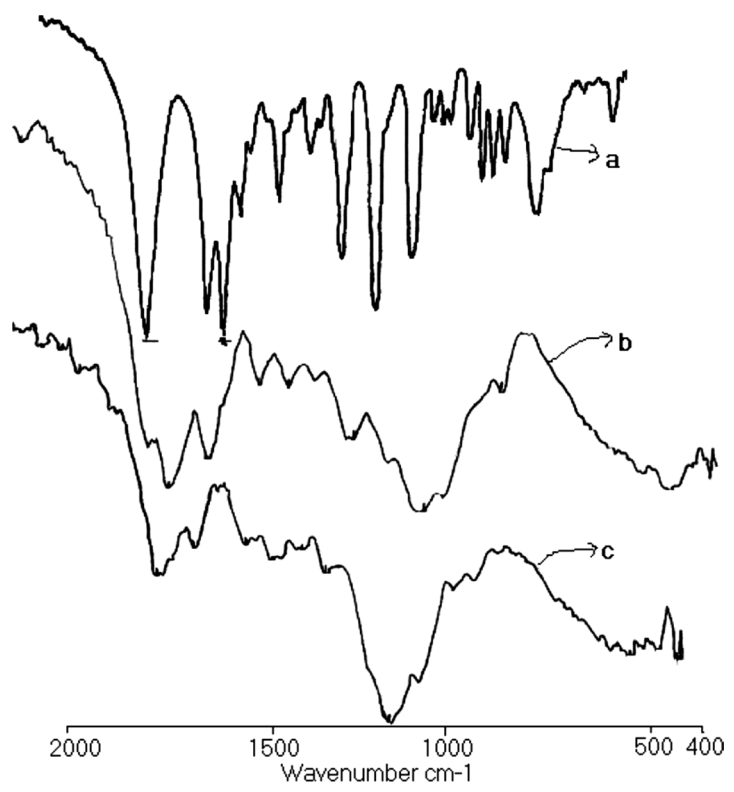

Fig. 2. FT-IR Spectra of (a) Lomustine (b) LMT Loaded CH Nanoparticles (c) PEG Coated LCNPs 

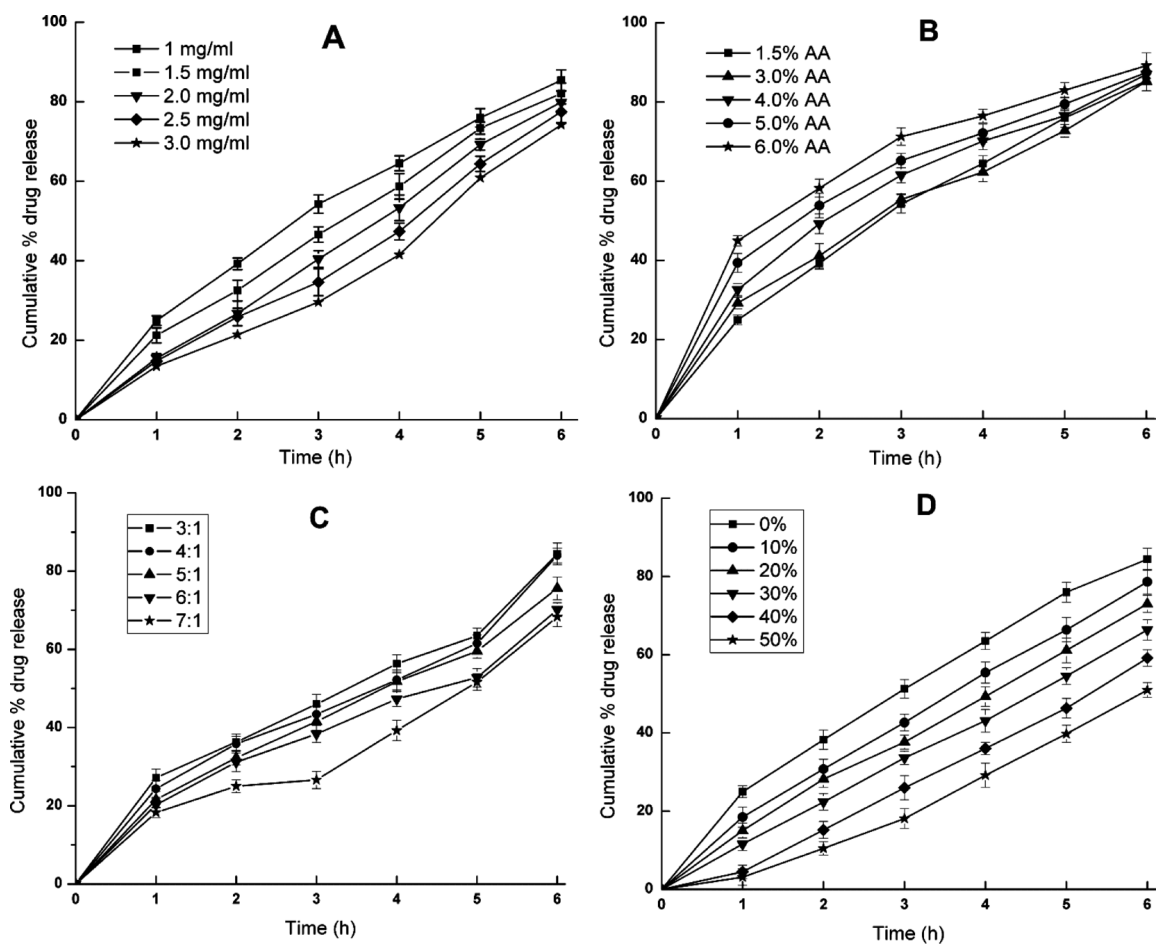

Fig. 3. In-Vitro Release Study of LCNPs (A) Effect of CH Concentration (B) Effect of Acetic Acid Concentration (C) Effect of CH : TPP Mass Ratio (D) Effect of PEG Concentration (Coating to LCNPs)

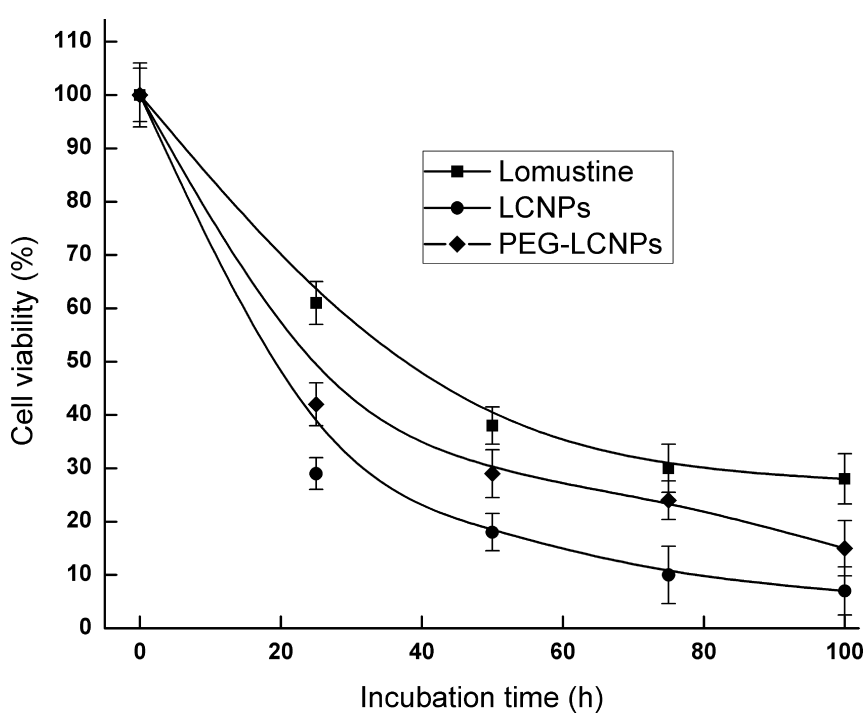

Fig. 4. In-Vitro Cytotoxic Activity (Viability of L132 Cells Incubated) of Lomustine, LCNPs and PEG-LCNPs

effect on the proliferation of the L132 cells were observed with LCNP. In the present study, the dependence of LCNPs cytotoxicity and cell viability might be related to the size and zeta potential of the nanoparticles. The particle size of PEG 6000 coated LCNPs (batch LCP1) appeared to be the dominant factor influencing the cytotoxicity of LCNPs. PEG coated LCNPs showed a significantly lower cytotoxicity than LCNPs as they were 6.5 fold larger in mean diameter. The reduction of cell viability also correlates well with the zeta potential data. The reduced cytotoxicity of PEG coated LCNPs compared with the LCNPs was in line with its zeta potential $(47.6 \mathrm{mV}$ vs. $49.3 \mathrm{mV})$. Cytotoxicity of LMT is in- cubation time and concentration dependent, but cell viability of LCNPs is more related to time of incubation as compared to concentration factor in LMT showing even at $48 \mathrm{~h}$. Thus, the LCNPs have better potency than free drug as have been reported earlier. ${ }^{15-17)}$

\section{Discussion}

All the formulations of LCNPs revealed particle size in nano range with positive zeta potential that makes suitable of therapeutic application LMT. In addition, the larger sized nanoparticles with higher $\mathrm{CH}$ concentration may also contribute to a higher amount of unneutralized $-\mathrm{NH}^{3+}$ led to stretching of the $\mathrm{CH}$ chain to result in larger nanoparticles. $^{18,19)}$ Consequently, higher amounts of TPP with constant mass of $\mathrm{CH}$ could saturate the cationic sites of the polymeric chain and increase the size of the nanoparticles. The change in concentration of acetic acid changes the solubility of $\mathrm{CH}$ in aqueous medium slightly. Its acidic $\mathrm{pH}$ decreases from 5.0 to 3.8 by increasing the concentration of acetic acid which in turn causes early precipitation of $\mathrm{CH}$ upon contact with basic TPP solution ( $\mathrm{pH}$ 8.3) due to increased ionization of amine group proceeding to stronger cross-linking density and formation of a polymeric wall at a shorter distance in between the nanodroplets associated with a decrease in the resultant chitosan NPs size. This smaller particle size due to addition of increasing concentration of acetic acid exhibited higher drug release rates. Due to a corresponding increase in the total $\mathrm{CH}$ NP surface area resulting in a larger drug being fraction exposed to the leaching medium.

Increase in size and reduced in zeta potential of fabricated PEG coated CH nanoparticles is good indication of association of PEG and $\mathrm{CH}$ nanoparticles. The interaction between oxygen atom of PEG and amino group of $\mathrm{CH}$ is weak, that attributed larger size of PEG coated nanoparticles compared 
to $\mathrm{CH}$ nanoparticles. ${ }^{20,21)}$ The differences in particle size observed in TEM, SEM and Zetasizer might be attributed to the swelling of $\mathrm{CH}$ in presence of water.

The increasing the encapsulation efficiency with increase PEG concentration may be due to surface and intercalation of LMT in between the PEG and CH. Therefore, a mixed interfacial film was formed at the interface that resulted in decreased positive surface charge. This decrease in the surface charge suggests the formation of stabilizing adsorbed polymer layer. On the other hand, increasing $\mathrm{CH}$ concentration led to an increase in the mean diameter with unexpected decrease in the positive zeta potential value. This can be solely attributed to the increase in the dispersion viscosity of $\mathrm{CH}$. Entrapment efficiency of the nanoparticles was affected by the LMT concentration in the $\mathrm{CH}$ solution and with increasing ratio of $\mathrm{CH}$ : TPP leading to a slight increase of entrapment efficiency. The mechanism of LMT association to chitosan NPs was mediated by an ionic interaction. ${ }^{22)}$ In the present study, the observed increase in entrapment efficiency with increasing $\mathrm{CH}$ concentration may be due to adsorption and electrostatic attraction of LMT that was confirmed by FT-IR, which showed hydrogen bond formation between hydroxyl group of $\mathrm{CH}$ and carbonyl group of LMT as there was shift of amide-carbonyl at $1703 \mathrm{~cm}^{-1}$ of LMT to $1650 \mathrm{~cm}^{-1}$.

Increased stretching effect due to increased shearing speed of homogenizer decreased the size of the resulting nanoparticles, ${ }^{23)}$ It indicated that the shear stress of homogenization onto the reaction solution during the gelation process facilitates the formation of fine particle. This may be due to difference in degradation mechanisms of $\mathrm{CH}$ molecules and different size of degraded $\mathrm{CH}$ molecules participating in the ionotropic gelation with TPP molecules. Much more $\mathrm{CH}$ debris and smaller degraded molecules were observed due to stretching effect exerted by mechanical shearing. Smaller fragments of $\mathrm{CH}$ resulted in lesser ionotropic gelation of CH-TPP nanoparticles. ${ }^{24)}$

As observed by in vitro release data, PEG coated LCNPs resulted in the slow release of LMT in comparison with that of LCNPs within $6 \mathrm{~h}$, which indicated that even though introduction of PEG 6000 increased the hydrophilicity of $\mathrm{CH}$, entrapment efficiency was also increased and hydrophobic characteristics of entrapped LMT molecules resulted in slow release due to retarded drug diffusion. The overall release process of LMT was biphasic, with an initial burst effect, and followed by a leveling-off of the release phase. The burst release of LMT was associated with those LMT molecules dispersing close to the nanocarriers surface, which easily diffuse out in the initial incubation time in addition, large specific surface area that characterized nanocarriers can adsorb the drug so the first burst release is possibly due to part of the drug desorbed from nanoparticle surface. ${ }^{2,25)}$ Later, LMT was released slowly after swelling and degradation of the polymer. The in-vitro release data of batches (LC1, LC6, LC7) that contained different amounts of LMT, released amount depended on the entrapment efficiency of nanoparticles prepared from the same material. As expected, the higher the entrapment efficiency the less LMT was released. A significant burst release was observed from the higher entrapment efficiency nanoparticles, which is due to more LMT adsorption on the surface of nanoparticles, leading to a faster release rate in the burst phase. In the leveling-off release phase how- ever, a similar release rate was observed with the variation in entrapment efficiency. This indicates that diffusion pathway plays an important role at this stage, which led to decreased concentration gradient (the driving forces for diffusion). Consequently, these results indicate that is possible to modulat the release of LMT by regulating the entrapped amount. The kinetic model that governed the overall release profile of LMT from the prepared nanocarriers in phosphate buffer sink solution was the diffusion of the drug through the thin polymeric membrane. The release data was kinetically analyzed using the empirical equation: $Q_{t}=K_{\mathrm{H}} \sqrt{t}$ (Higuchi square root model). Based on the diffusion exponent, the nanoparticle formulae revealed $n$-values of $(1>n>0.5)$, meaning non-Fickian diffusion behavior that was obtained as a result of contribution from diffusion. ${ }^{10)}$

Previously, cellular uptake of polymer nanoparticles and ultimate reduction of cell viability have been reported, influenced by particle size. ${ }^{26,27)}$ Zang et al. ${ }^{28)}$ indicated that the cellular uptake is strongly dependent on the particle size. Particle size of nanoparticles has been reported to play an important role in their antitumor activity and thus the cytotoxic activity of the chitosan nanoparticles increased with decreasing particle size. Also the greater the zeta potential of the nanoparticles, leads to a stronger interaction with tumor cell membrane, and leads to higher cytotoxicity. ${ }^{29)}$

\section{Conclusion}

Chitosan nanoparticles fabricated by a slightly modified ionotropic gelation method showed an excellent capacity for the association of LMT and the polymer. The different parameters such as mechanical shearing, chitosan concentrations, acidic environment, $\mathrm{CH}$ : TPP ratio and LMT amount significantly affected the particle size, zeta potential and drug encapsulation. The characterization of particles size of nanoparticles was supported by DLS, SEM and TEM images. In-vitro release study of drug was diffusion controlled over the $6 \mathrm{~h}$. The in-vitro cytotoxicity of optimized formulation in lung cancer cell line showed a significant effect.

Acknowledgements We acknowledge the generosity of Marine Chemicals (Cochin, Kerala) and Devdhar chemicals (Pune) for providing the gift samples of chitosan and TPP.

\section{References}

1) Xang X. G., Teng D. Y., Wu Z. M., Wang X., Wang Z., Yu D. M., Li C. X., J. Mater. Sci. Mater. Med., 19, 3525-3533 (2008).

2) Agnihotri S. A., Mallikarjuna N. N., Aminabhavi T. M., J. Controlled Release, 100, 5-28 (2004).

3) Sarmento B., Ferreira D., Veiga F., Ribeiro A., Carbohydr. Polym., 66, $1-7$ (2006).

4) Vila A., Sanchez A., Janes K., Behrens I., Kissel T., Jato J. L. V., Alonso M. J., Eur. J. Pharm. Biopharm., 57, 123-131 (2004).

5) Yuan X. B., Li H., Yuan Y. B., Carbohydr. Polym., 65, 337-345 (2006).

6) Lee D. W., Powers K., Baney R., Carbohydr. Polym., 58, 371-377 (2004).

7) Lee S. T., Mi F. L., Shen Y. J., Shyu S. S., Polymer, 42, 1879-1892 (2001).

8) Shu X. Z., Zhu K. J., Int. J. Pharm., 201, 51-58 (2000),

9) Tang E. S. K., Huang M., Lim L. Y., Int. J. Pharm., 265, 103-114 (2003).

10) Tsai M. L., Bai S. W., Chen R. H., Carbohydr. Polym., 71, 448-457 (2008).

11) Gerf R., Minamitake Y., Perracchia M. T., Trubetskoy V., Torchilin V., Langer R., Science, 263, 1600-1603 (1994).

12) Otsuka H., Nagasaki Y., Kataoka K., Adv. Drug Deliv. Rev., 55, 403 
419 (2003)

13) Hu Y., Jiang X., Ding Y., Zhang L., Yang C., Zhang J., Chen J., Yang Y., Biomaterials, 24, 2395-2404 (2003).

14) Lopezleon T., Carvalho E., Seijo B., Ortegavinuesa J., Bastosgonzalez B., J. Colloid Interface Sci., 283, 344-351 (2005).

15) Zhang S., Li J., Lykotrafitis G., Bao G., Suresh S., Adv. Mater, 21, $419-424$ (2009).

16) Nasti A., Zaki N. M., Leonardis P., Pharm. Res., 26, 1918-1930 (2009).

17) Trickler W., Nagvekar A., Dash A., Pharm. Res., 26, 1963-1973 (2009).

18) Nagarwal R. C., Kant S., Singh P. N., Maiti P., Pandit J. K., J. Controlled Release, 136, 2-13 (2009).

19) Nagarwal R. C., Singh P. N., Kant S., Maiti P., Pandit J. K., Chem. Pharm. Bull., 59, 272-278 (2011)

20) Kim S. S., Lee Y. M., Macromer. Polym., 36, 4494- 4501 (1995).

21) Wu Y., Yang W., Wang C., Hu J., Fu S., Int. J. Pharm., 295, 235-245
(2005).

22) Pan Y., Li Y. J., Zhao H. Y., Zheng J. M., Xu H., Wei G., Hao J. S., Cui F. D., Int. J. Pharm., 249, 139-147 (2002).

23) Zhang X. G., Teng D. Y., Wu Z. M., Wang X., Wang Z., Yu D. M., Li C. X., J. Mater. Sci.: Mater. Med., 19, 3525-3533 (2008).

24) Gan Q., Wang T., Cochrane C., McCrron P., Colloids Surf. B Biointerfaces, 44, 65-73 (2005).

25) Xu Y., Du Y., Int. J. Pharm., 250, 215-226 (2003).

26) Jung T., Kamm W., Breitenbach A., Kaiserling E., Xiao J. X., Kissel T., Eur. J. Pharm. Biopharm., 50, 147-160 (2000).

27) Jani P., McCarthy D. E., Florence A. T., Int. J. Pharm., 86, 239-246 (1992).

28) Zhang S., Li J., Lykotrafitis G., Bao G., Suresh S., Adv. Mater, 21, 419-424 (2009).

29) Qi L., Xu Z., Jiang X., Li Y., Wang M., Bioorg. Med. Chem. Lett., 15, 1397-1399 (2005). 